\title{
Enhancing Photocatalytic Degradation of Methyl Blue Using PVP-Capped and Uncapped CdSe Nanoparticles
}

\author{
Kgobudi Frans Chepape, Thapelo Prince Mofokeng, Pardon Nyamukamba, \\ Kalenga Pierre Mubiayi, and Makwena Justice Moloto \\ Department of Chemistry, Vaal University of Technology, Private Bag X021, Vanderbijlpark 1900, South Africa \\ Correspondence should be addressed to Kalenga Pierre Mubiayi; pierremub@gmail.com
}

Received 29 June 2017; Revised 27 September 2017; Accepted 11 October 2017; Published 6 December 2017

Academic Editor: Said M. El-Sheikh

Copyright (c) 2017 Kgobudi Frans Chepape et al. This is an open access article distributed under the Creative Commons Attribution License, which permits unrestricted use, distribution, and reproduction in any medium, provided the original work is properly cited.

Quantum confinement of semiconductor nanoparticles is a potential feature which can be interesting for photocatalysis, and cadmium selenide is one simple type of quantum dot to use in the following photocatalytic degradation of organic dyes. CdSe nanoparticles capped with polyvinylpyrrolidone (PVP) in various concentration ratios were synthesized by the chemical reduction method and characterized. The transmission electron microscopy (TEM) analysis of the samples showed that $50 \%$ PVP-capped CdSe nanoparticles were uniformly distributed in size with an average of $2.7 \mathrm{~nm}$ and shape which was spherical-like. The photocatalytic degradation of methyl blue (MB) in water showed efficiencies of $31 \%$ and $48 \%$ when using uncapped and $50 \%$ PVP-capped CdSe nanoparticles as photocatalysts, respectively. The efficiency of PVP-capped CdSe nanoparticles indicated that a complete green process can be utilized for photocatalytic treatment of water and waste water.

\section{Introduction}

Nanotechnology provides new avenues for the advancement of current technologies and has the potential to touch all areas of applications including health, environmental, and economic issues [1-3]. Environmental concerns are found everywhere in the world, and water treatment is an important aspect of life. Various contaminants can be found in water among which are organic pollutants that are of serious concern [4-6]. Nanoparticles possess new features such as size tunability and new mechanisms which are part of promising technology. Therefore, nanoparticles can be used as photocatalysts to facilitate the degradation of organic pollutants such as dyes upon UV-Vis irradiation [6-8].

There are different types of nanoparticles that can be used for the photodegradation of organic pollutants. Titanium oxide and zinc oxide have been given much attention in photocatalysis because of their availability, and they were known for centuries [9-12]. There are so many investigations done in order to improve the performance of $\mathrm{TiO}_{2}$ as a catalyst. Other types of materials are also investigated with attempts to bring new features $[5,6,8,12]$. CdSe nanoparticles with tuned band gap energy can be synthesized and may play an important role in photocatalysis besides other applications such as laser diodes, catalysis, light-emitting diodes, solar cells, and biological labeling [13]. Different methods for preparation of CdSe nanoparticles such as hydrothermal methods have been established in order to investigate the photocatalytic activity on degradation of organic pollutants [14].

In this study, CdSe nanoparticles were synthesized using PVP as a capping agent at different concentrations at room temperature. The capped and uncapped CdSe nanoparticles were characterized and tested for their photocatalytic activities using methylene blue dye as a model organic pollutant.

\section{Methodology}

2.1. Chemicals. Sodium borohydride, selenium powder, cadmium chloride, polyvinylpyrrolidone (PVP, Mw 40,000), methylene blue $(\mathrm{MB})$, and methanol were purchased from Sigma-Aldrich. All the chemicals were of analytical grade, and they were used as purchased. 


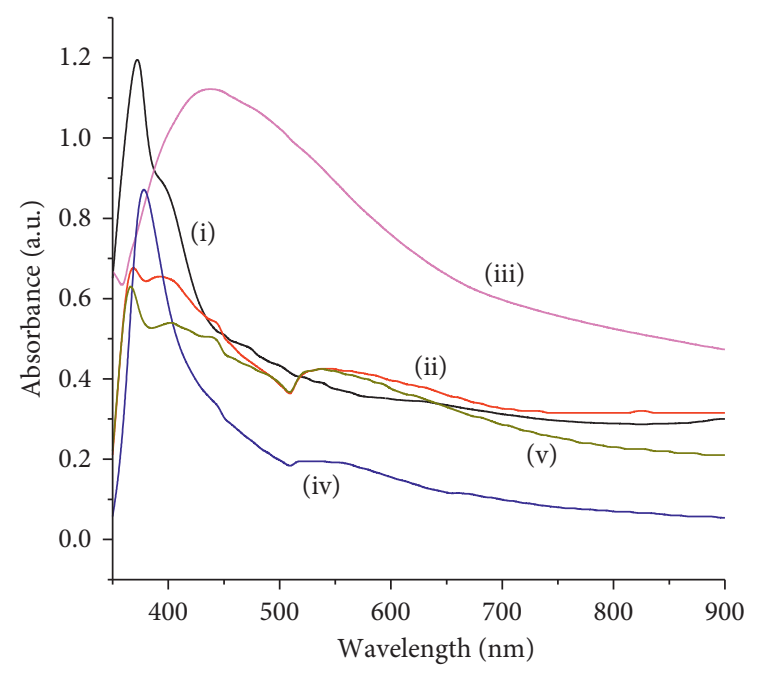

(a)

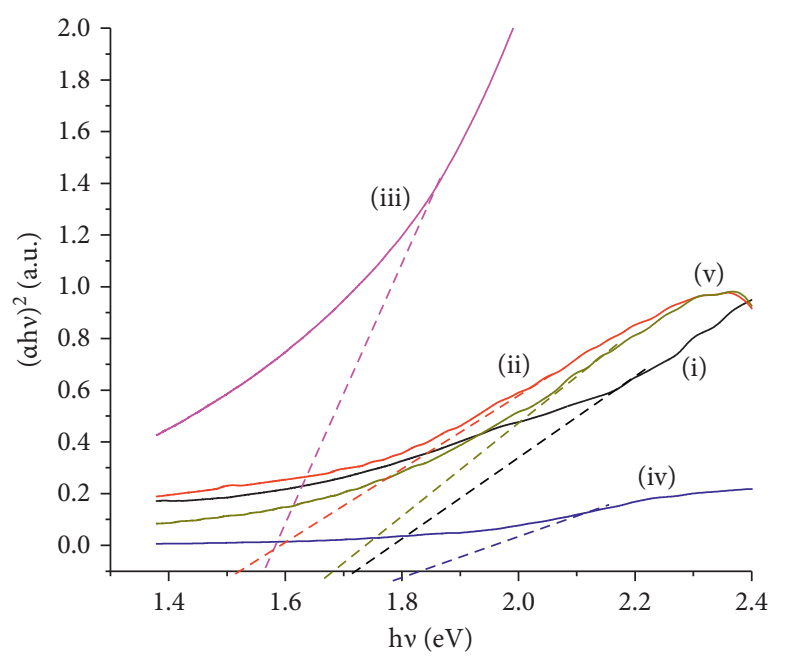

(b)

FIgUre 1: (a) Absorption spectra of (i) uncapped and (ii-v) PVP-capped CdSe nanoparticles with (b) their corresponding Tauc plots.

2.2. Synthesis of CdSe Nanoparticles. For a typical synthesis of uncapped CdSe, $25.3 \mathrm{mg}$ of selenium powder $(0.320 \mathrm{mmol})$ was placed in a $100 \mathrm{~mL}$ three-necked round-bottom flask which was then put under nitrogen atmosphere. The selenium was reduced by the addition of a $20.0 \mathrm{~mL}$ solution of sodium borohydride $(0.320 \mathrm{mmol})$ while stirring. The reaction was allowed to proceed for 3 hours in order to allow complete reduction of selenium. A $20.0 \mathrm{~mL}$ solution of $\mathrm{CdCl}_{2}(0.320 \mathrm{mmol})$ was added to the flask content which was kept stirring for an hour at room temperature. The nanoparticles were washed using a centrifuge (5000 rpm for $10 \mathrm{~min}$ each cycle) with methanol and dried in air for 48 hours. The PVP-capped CdSe nanoparticles were synthesized using the same procedure as that of uncapped CdSe nanoparticles, but $0.320 \mathrm{mmol}$ of $\mathrm{CdCl}_{2}$ was simultaneously added with $20.0 \mathrm{~mL}$ of PVP (1.288 g). Four different PVP concentrations were considered by varying the mass of PVP. Thus, $1 \%, 25 \%, 50 \%$, and 75\% PVP-capped CdSe nanoparticles were synthesized.

2.3. Photocatalytic Activity Evaluation. The photocatalytic activities of uncapped and PVP-capped CdSe nanoparticles were evaluated using the $\mathrm{MB}$ dye as a model pollutant under UV light set at $12 \mathrm{~W}$ in a photoreactor equipped with a $12 \mathrm{~W}$ UV lamp and a peristaltic pump. A peristaltic pump was used for continuous air flow to ensure uniform mixing of the catalyst and solution in the reactor. All the photodegradation experiments were conducted at $25^{\circ} \mathrm{C}$. Typically, $5 \mathrm{mg}$ of the CdSe nanoparticles were added to $50 \mathrm{~mL}$ of $10 \mathrm{ppm} \mathrm{MB}$ aqueous solution in a $100 \mathrm{~mL}$ beaker. The beaker was covered with an aluminium foil and stirred magnetically in the dark for 30 minutes for adsorption and desorption equilibrium to take place. After 30 minutes of stirring, $5 \mathrm{~mL}$ of the aliquot was collected as the initial mark ( 0 min exposure) for absorption measurement by a UV-Vis spectrometer. Meanwhile, the remaining solution was inserted into a $250 \mathrm{~mL}$ photoreactor. To detect the changes in $\mathrm{MB}$ concentration with time, aliquots were collected at
$5,10,20,30,60$, and $120 \mathrm{~min}$ and then analyzed by the $\mathrm{UV}-\mathrm{V}$ is spectrometer.

2.4. Characterization. The absorption measurements were performed at room temperature using a PerkinElmer Lambda $25 \mathrm{UV}-\mathrm{V}$ is spectrometer with a wavelength range from $200 \mathrm{~nm}$ to $1100 \mathrm{~nm}$. The FTIR spectra were collected from a PerkinElmer spectrum 400 FTIR/NIR spectrometer with the wavenumber range of $650 \mathrm{~cm}^{-1}-4000 \mathrm{~cm}^{-1}$, and the analysis was done at room temperature. A Bruker D2 Phaser X-ray diffractometer was used to determine the crystalline properties of the nanoparticle's powder. The morphology of the synthesized CdSe nanoparticles was determined using a JEOL JEM-2100 transmission electron microscope operating at $200 \mathrm{KV}$.

\section{Results and Discussion}

Amongst semiconductor nanoparticles, regularly prepared cadmium selenide makes a better choice for its good properties because of its low band gap, and the generated electrons have high negative reduction potentials. It can be easily prepared using various methods including less energetic conditions such as employing a reducing agent, sodium borohydride, at room temperature. Cadmium selenide has a bulk band edge of nearly $730 \mathrm{~nm}$ in the visible region which can be tuned towards the $\mathrm{UV}$ region by controlling the particle size.

3.1. UV-Vis Spectral Analysis. UV-Vis absorption spectra of all synthesized CdSe nanoparticles are shown in Figure 1(a). The absorption peaks of the uncapped and capped CdSe nanoparticles were all below $600 \mathrm{~nm}$. All absorption band edges were blue-shifted between 500 and $600 \mathrm{~nm}$, suggesting that the synthesized nanoparticles were relatively small. The inflection point around $500 \mathrm{~nm}$ was due to the drift in the measurements. The change in absorption band edge is indicative of the different types of nanoparticles obtained from the synthesis with 

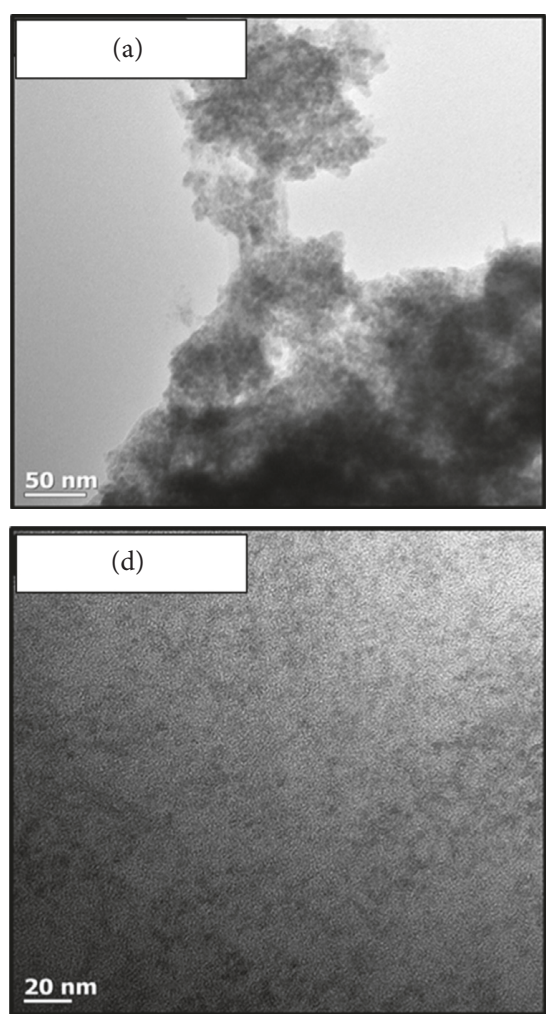
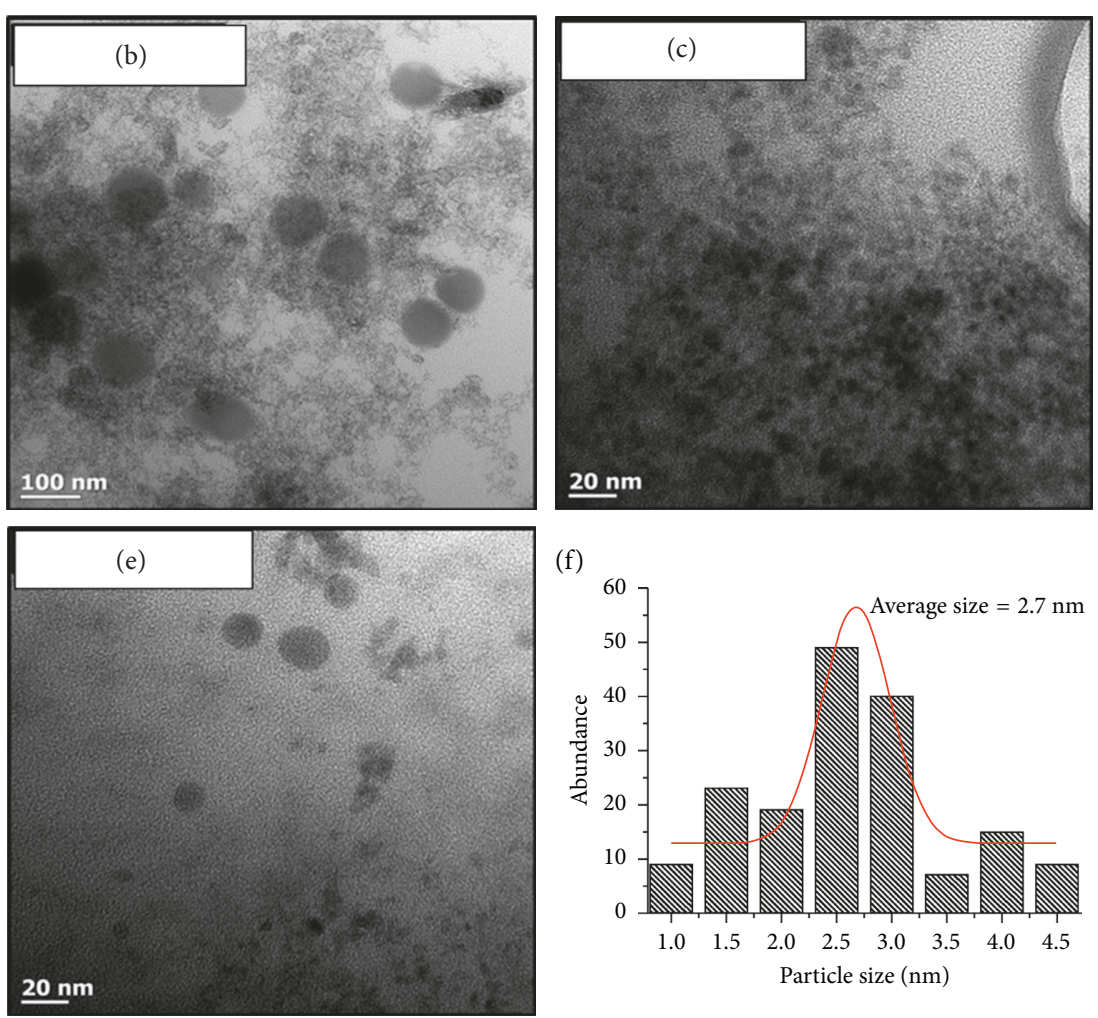

(f)

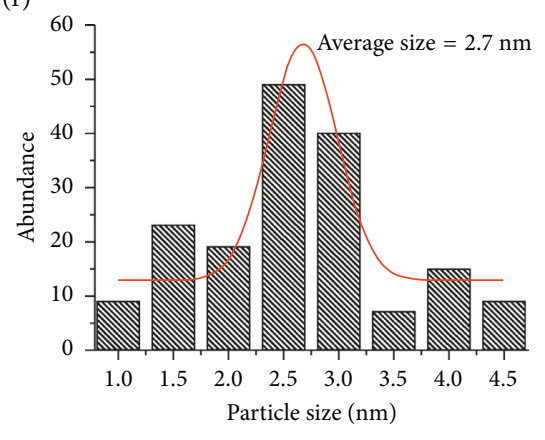

Figure 2: TEM images of (a) uncapped and (b) 1\%, (c) 25\%, (d) 50\%, and (e) 75\% PVP-capped CdSe nanoparticles, and (f) the size histogram of 50\% PVP-capped CdSe nanoparticles.

and without PVP. The difference might also be due to a change in particle distribution and/or dominance of defects. The smaller tailing in the spectrum of 50\% PVP-capped CdSe nanoparticles is indicative of small and well-dispersed particles.

The Tauc plots are shown in Figure 1(b). The approximation of band gap values was found in the range of 1.52$1.82 \mathrm{eV}$ for all the synthesized CdSe nanoparticles. The 50\% PVP-capped CdSe nanoparticles gave the highest energy in absorption band edges as compared to other percentages of PVP reported in this work.

3.2. TEM Analysis. The TEM images of the synthesized CdSe nanoparticles are shown in Figure 2. The formation of the particles differed as the precursor was uncapped or capped at different concentrations of PVP. The uncapped particles were agglomerated with a mixture of spherical-, dendritic-, and flower-like shapes and sizes compared to PVP-capped CdSe nanoparticles. The 1\% PVP-capped CdSe nanoparticles showed a mixture of bigger and smaller sphericallike nanoparticles. These structures tended to decrease to uniform and small features as the concentration of PVP increased. At 50\% PVP capping, the nanoparticles became well dispersed and uniformly distributed as shown in Figure 2(f). The average size distribution of these particles was $2.7 \mathrm{~nm}$, while the largest particles had a diameter of $4.5 \mathrm{~nm}$ in this sample. However, for PVP concentration above $50 \%$, the Ostwald ripening effect favored bigger particles as reported also by other authors [15-18]. Mahmoud and

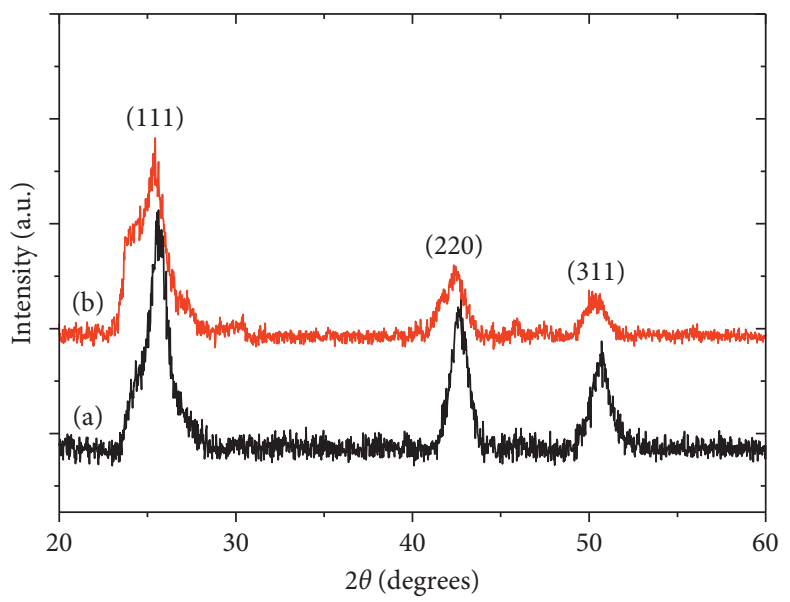

FIGURE 3: XRD pattern of (a) uncapped and (b) 50\% PVP-capped CdSe nanoparticles.

El-Mallah [15] employed PVP as a capping ligand at various concentrations for the synthesis of CdSe nanoparticles. PVPcapped CdSe nanoparticles which consisted of less agglomerated cubic-like structures with an average size of $2.3 \mathrm{~nm}$ were reported and were successfully used for photovoltaic application.

3.3. XRD Analysis. The X-ray diffraction patterns of $50 \%$ PVP-capped and uncapped CdSe nanoparticles are shown in Figure 3 . The synthesized nanoparticles relatively showed 


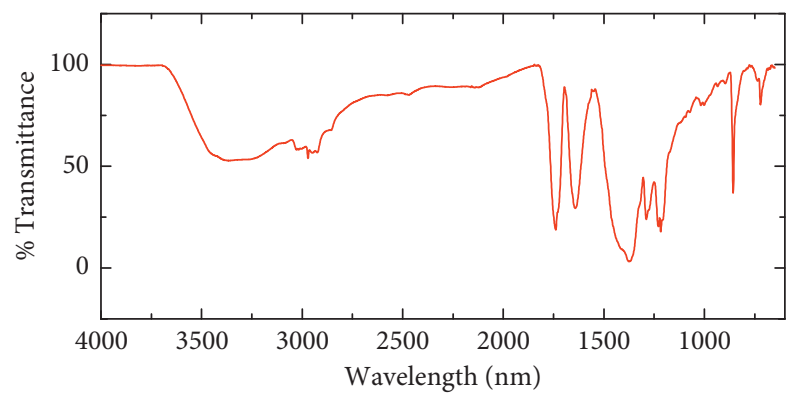

(a)

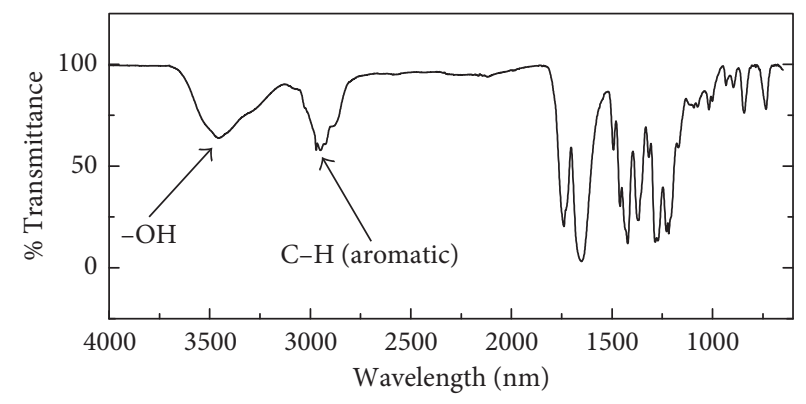

(b)

FIGURE 4: FTIR spectra of (a) 50\% PVP-capped CdSe nanoparticles and (b) pure PVP.

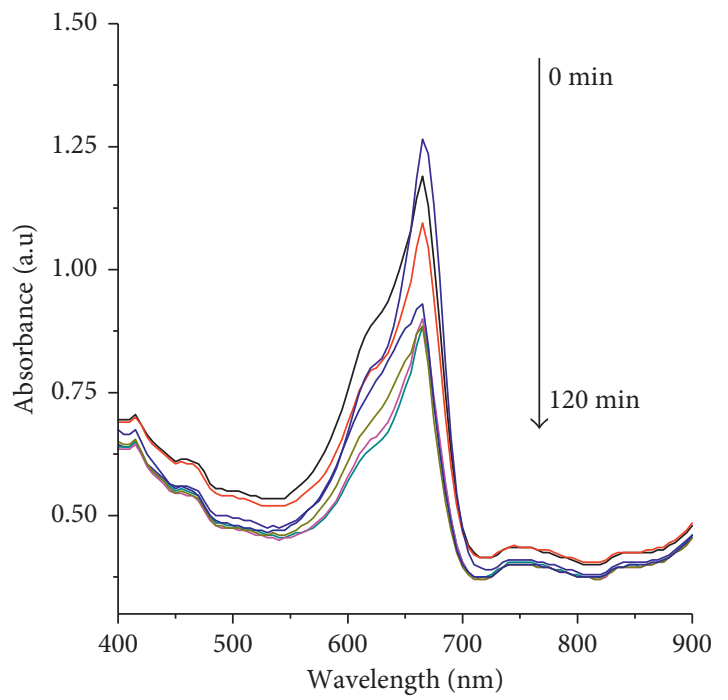

(a)

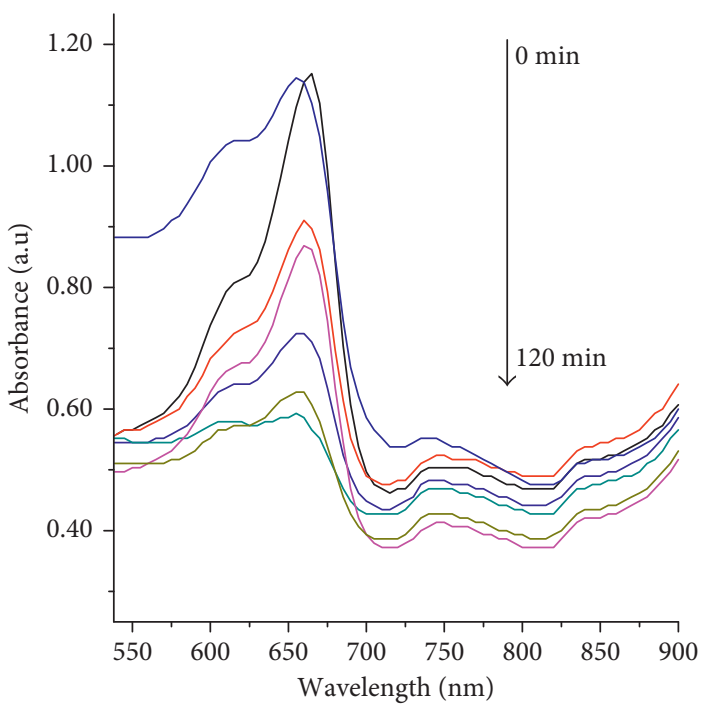

(b)

FIGURE 5: Absorption spectra of MB after UV light exposure in the presence of (a) uncapped and (b) 50\% PVP-capped CdSe nanoparticles.

some degree of crystallinity, and all major peaks with $2 \theta$ values around $25^{\circ}, 42^{\circ}$, and $50^{\circ}$ matched with the standard pattern of the cubic crystal of CdSe (JCPDS 19-0191) [19]. No peaks due to impurities were found. More peak defects were observed in the XRD pattern of PVP-capped CdSe nanoparticles as attested by appearance of more shoulders. This may be the result of structural modifications such as shape defects and sizes. A slight shift of about $0.3^{\circ}$ in the peak position towards lower $2 \theta$ values was observed in the pattern of PVP-capped CdSe nanoparticles in comparison with that of the uncapped material. This may be indicative of strain caused by the binding of PVP on the surface of nanoparticles.

3.4. FTIR Spectral Analysis. The FTIR spectral analysis of the PVP-capped CdSe nanoparticles was done to confirm the capping effect. The 50\% PVP-capped CdSe sample was particularly chosen for this analysis due to its optical properties. The peaks in the spectrum of pure PVP are similarly found in that of 50\% PVP-capped CdSe nanoparticles as shown in Figure 4. A slight shift in several peaks can be attributed to the binding of PVP to CdSe nanoparticles. The broad peak in the
-OH region confirmed the binding of PVP to CdSe nanoparticles where a peak shift was observed at $3250 \mathrm{~cm}^{-1}$ to $3540 \mathrm{~cm}^{-1}$. A shift in $\mathrm{C}-\mathrm{H}$ aromatic stretching was also observed in the region of $3137 \mathrm{~cm}^{-1}$ to $3201 \mathrm{~cm}^{-1}$ upon binding to CdSe nanoparticles, indicating the interference in hydrogen bonding due to interactions with cadmium ions and successive formation of CdSe nanoparticles. This corroborates the crystalline properties observed from XRD analysis.

3.5. Photocatalytic Activity Evaluation. The PVP-capped CdSe nanoparticles with varied percentages gave the optimum conditions when PVP concentration was $50 \%$. This was due to the largest absorption band edge energy, good particle size distribution, and smaller sizes of uniform shapes. Therefore, this sample was used to evaluate the photocatalytic activity in comparison with the uncapped CdSe nanoparticles. The photocatalytic degradation was performed by exposing the $\mathrm{MB}$ aqueous solution to the UV light in the presence of uncapped and 50\% PVP-capped CdSe nanoparticles for 0, 5, 10, 20, 30, 60, and $120 \mathrm{~min}$. The corresponding absorption spectra are shown in Figure 5. The absorption peak intensities 


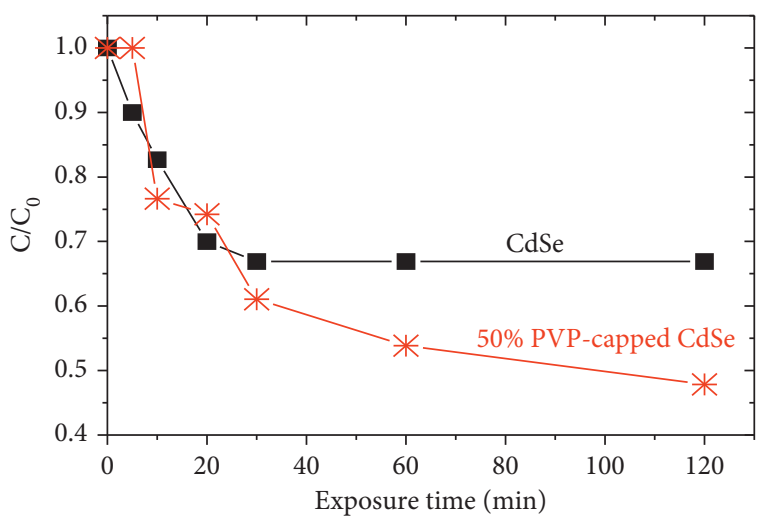

FIgURE 6: Photodegradation curves of $\mathrm{MB}$ concentration as function of time using uncapped and 50\% PVP-capped CdSe nanoparticles.

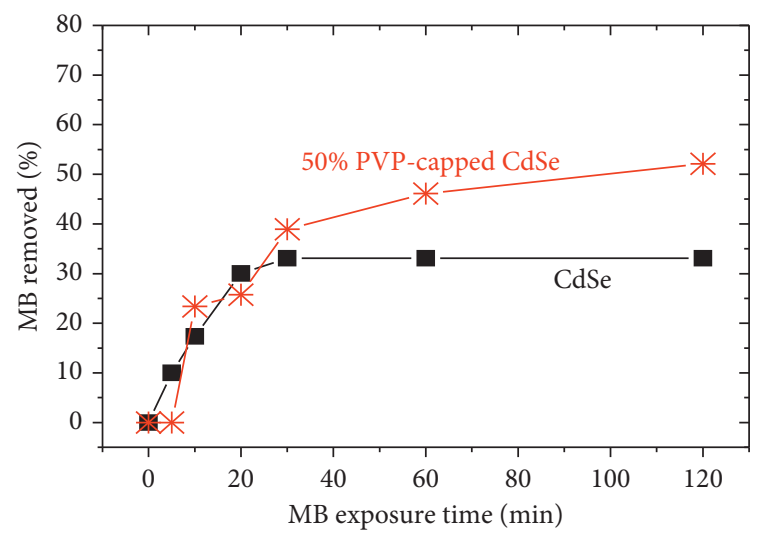

Figure 7: MB removal efficiency of uncapped and 50\% PVPcapped CdSe nanoparticles as function of UV light exposure time.

decreased as the exposure time increased for both uncapped and 50\% PVP-capped CdSe samples. This is indicative of the change in amounts of $\mathrm{MB}$ with exposure time. The ratio of concentration of $\mathrm{MB}$ remaining to the initial one $\left(\mathrm{C} / \mathrm{C}_{0}\right)$ over time is depicted in Figure 6. The photodegradation was confirmed since the initial amount of $\mathrm{MB}$ was not the same after exposure to UV irradiation for both uncapped and 50\% $\mathrm{PVP}$-capped CdSe samples. The ratio $\mathrm{C} / \mathrm{C}_{0}$ decreased when $50 \%$ PVP-capped CdSe nanoparticles were used. The $\mathrm{C} / \mathrm{C}_{0}$ value was less than 0.50 compared to that of the uncapped CdSe nanoparticles which had a value of 0.65 .

The MB removal efficiency increased gradually with time until the optimum activity was achieved for both samples, after which no major variation could be observed as shown in Figure 7. The uncapped CdSe nanoparticles degraded $31 \%$ of MB in water after 2 hours, whilst the 50\% PVP-capped CdSe nanoparticles degraded $48 \%$ of MB in water after the same exposure time. This indicates that the properties of the capped and uncapped CdSe nanoparticles were not identical as also seen in their structures and optical behaviour. The controlled size and surface area of nanoparticles capped with PVP are the main properties affecting the photocatalytical performance.

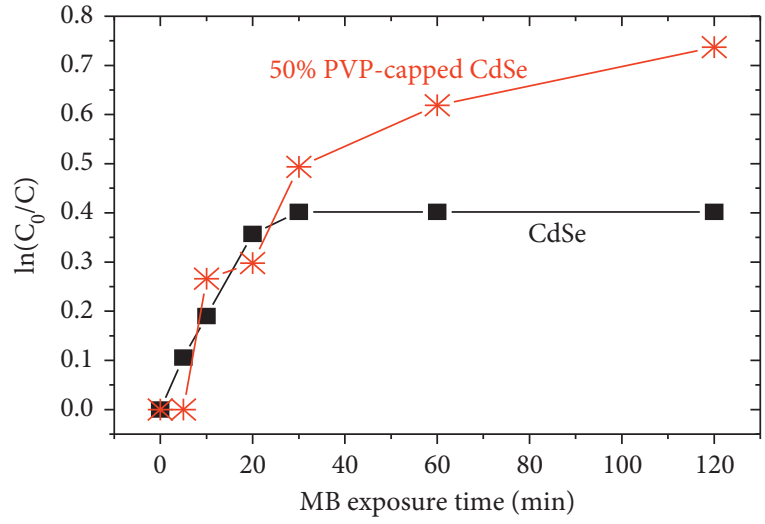

FIGURE 8: Kinetic studies of photodegradation of $\mathrm{MB}$ in the presence of uncapped and 50\% PVP-capped CdSe nanoparticles.

The nanoparticles prepared using PVP as a capping agent exhibit better optical and structural properties due to controlled growth. Haldar et al. [20] used pure CdSe nanocrystals and $\mathrm{Au} / \mathrm{CdSe}$ heterostructures as catalysts for the degradation of R6G in water. They reported only $27.3 \%$ of R6G removal from water using CdSe, whilst the $\mathrm{Au} / \mathrm{CdSe}$ heterostructures allowed for $87.2 \%$ removal of the same dye under the same UV irradiation for $150 \mathrm{~min}$.

The kinetic studies are shown in Figure 8. The fitted straight lines (not shown) could not cover most of the points plotted in the $\ln \left(\mathrm{C}_{0} / \mathrm{C}\right)$ versus time curve, the R-squared values showed a poor fitting indicating a nonlinearity, and consequently, the degradation was not following the first-order kinetics.

\section{Conclusion}

The CdSe nanoparticles prepared gave relatively blue-shifted band edges with smaller particle sizes using PVP as a capping molecule at all percentages. XRD confirmed that CdSe nanoparticles were synthesized using PVP as a capping agent. The 50\% PVP-capped CdSe nanoparticles had relatively improved structural and optical properties and showed uniform distribution and hence chosen for the photodegradation of methylene blue. The 50\% PVP-capped CdSe nanoparticles demonstrated a better photocatalytic degradation of methylene blue in water compared to the uncapped CdSe nanoparticles. The amount of MB to be utilized for water samples should be optimized further for $100 \%$ photocatalytic activity. In other respects, the UV-Vis irradiation power should be studied further in order to have optimized effect of the photocatalyst. Moreover, the synthesized nanoparticles can be utilized in many applications.

\section{Conflicts of Interest}

The authors declare that they have no conflicts of interest.

\section{Acknowledgments}

The authors are thankful to the National Research Foundation in South Africa and Vaal University of Technology for funding and facilities. 


\section{References}

[1] R. Cai, Y. Kubota, T. Shuin, H. Sakai, K. Hashimoto, and A. Fujishima, "Induction of cytotoxity by photoexcited $\mathrm{TiO}_{2}$ particles," Cancer Research, vol. 52, no. 8, pp. 2346-2348, 1992.

[2] M. Hainlaan, A. Ivask, I. Blinova, H. C. Dubourguier, and A. Kahru, "Toxicity of nanosized and bulk $\mathrm{ZnO}, \mathrm{CuO}$ and $\mathrm{TiO}_{2}$ to bacteria Vibrio fischeri and crustaceans Daphnia magna and Thamnocephalus platyurus," Chemosphere, vol. 71, no. 7, pp. 1308-1316, 2008.

[3] P. Ball, "Natural strategies for the molecular engineer," Nanotechnology, vol. 13, no. 5, pp. R15-R28, 2002.

[4] T. He, H. Ma, Z. Zhou et al., "Preparation of ZnSFluoropolymer nanocomposites and its photocatalytic degradation of methylene blue," Polymer Degradation and Stability, vol. 94, no. 12, pp. 2251-2256, 2009.

[5] X. Wang, G. Liu, G. Q. Lu, and H.-M. Cheng, "Stable photocatalytic hydrogen evolution from water over $\mathrm{ZnO}-\mathrm{CdS}$ core-shell nanorods," International Journal of Hydrogen Energy, vol. 35, no. 15, pp. 8199-8205, 2010.

[6] K. Simeonidis, S. Mourdikoudis, E. Kaprara, M. Mitrakasd, and L. Polavarapu, "Inorganic engineered nanoparticles in drinking water treatment: a critical review," Environmental Science: Water Research \& Technology, vol. 2, no. 1, pp. 43-70, 2016.

[7] I. Gehrke, A. Geiser, and A. Somborn-Schulz, "Innovations in nanotechnology for water treatment," Nanotechnology, Science and Applications, vol. 8, pp. 1-17, 2015.

[8] X. Qu, P. J. Alvarez, and Q. Li, "Applications of nanotechnology in water and wastewater treatment," Water Research, vol. 47, no. 12, pp. 3931-3946, 2013.

[9] Y. Abdollahi, A. H. Abdullah, Z. Zainal, and N. A. Yusof, "Photocatalytic degradation of p-cresol by zinc oxide under UV irradiation," International Journal of Molecular Sciences, vol. 13, no. 12, pp. 302-315, 2011.

[10] A. M. Tayeb and D. S. Hussein, "Synthesis of $\mathrm{TiO}_{2}$ nanoparticles and their photocatalytic activity for methylene blue," American Journal of Nanomaterials, vol. 3, no. 2, pp. 57-63, 2015.

[11] G. Tian, H. Fu, L. Jing, and C. Tian, "Synthesis and photocatalytic activity of stable nanocrystalline $\mathrm{TiO}_{2}$ with high crystallinity and large surface area," Journal of Hazardous Materials, vol. 161, no. 2-3, pp. 1122-1130, 2009.

[12] S. Ivanov, A. Barylyak, K. Besaha et al., "Synthesis, characterization, and photocatalytic properties of sulfur- and carbon-codoped $\mathrm{TiO}_{2}$ nanoparticles," Nanoscale Research Letters, vol. 11, no. 1, p. 140, 2016.

[13] T. Ghosh, J.-H. Lee, Z.-D. Meng et al., "Graphene oxide based CdSe photocatalysts: synthesis, characterization and comparative photocatalytic efficiency of rhodamine B and industrial dye," Materials Research Bulletin, vol. 48, no. 3, pp. 1268-1274, 2013.

[14] X. Liu, C. Ma, Y. Yan et al., "Hydrothermal synthesis of CdSe quantum dots and their photocatalytic activity on degradation of cefalexin," Industrial \& Engineering Chemistry Research, vol. 52, no. 43, pp. 15015-15023, 2013.

[15] W. E. Mahmoud and H. M. El-Mallah, "Synthesis and characterization of PVP-capped CdSe nanoparticles embedded in PVA matrix for photovoltaic application," Journal of Physics D: Applied Physics, vol. 42, no. 3, p. 035502, 2009.

[16] G. Murugadoss, "Synthesis and optical characterization of PVP and SHMP-encapsulated $\mathrm{Mn}^{2+}$-doped $\mathrm{ZnS}$ nanocrystals," Journal of Luminescence, vol. 130, no. 11, pp. 2207-2214, 2010.
[17] G. Ghosh, M. Kanti Naskar, A. Patra, and M. Chatterjee, "Synthesis and characterization of PVP-encapsulated ZnS nanoparticles," Optical Materials, vol. 28, no. 8-9, pp. 10471053, 2006.

[18] K. M. Koczkur, S. Mourdikoudis, L. Polavarapu, and S. E. Skrabalak, "Polyvinyl pyrrolidone (PVP) in nanoparticle synthesis," Dalton Transactions, vol. 44, no. 41, pp. 1788317905, 2015.

[19] Powder Diffraction Data File, Joint Committee of Powder Diffraction Standard, International Center for Diffraction Data, Newtown Square, PA, USA, Card No. 19-0191, 1999.

[20] K. K. Haldar, G. Sinha, J. Lahtinen, and A. Patra, "Hybrid colloidal Au-CdSe pentapod heterostructures synthesis and their photocatalytic properties," ACS Applied Materials \& Interfaces, vol. 4, no. 11, pp. 6266-6272, 2012. 

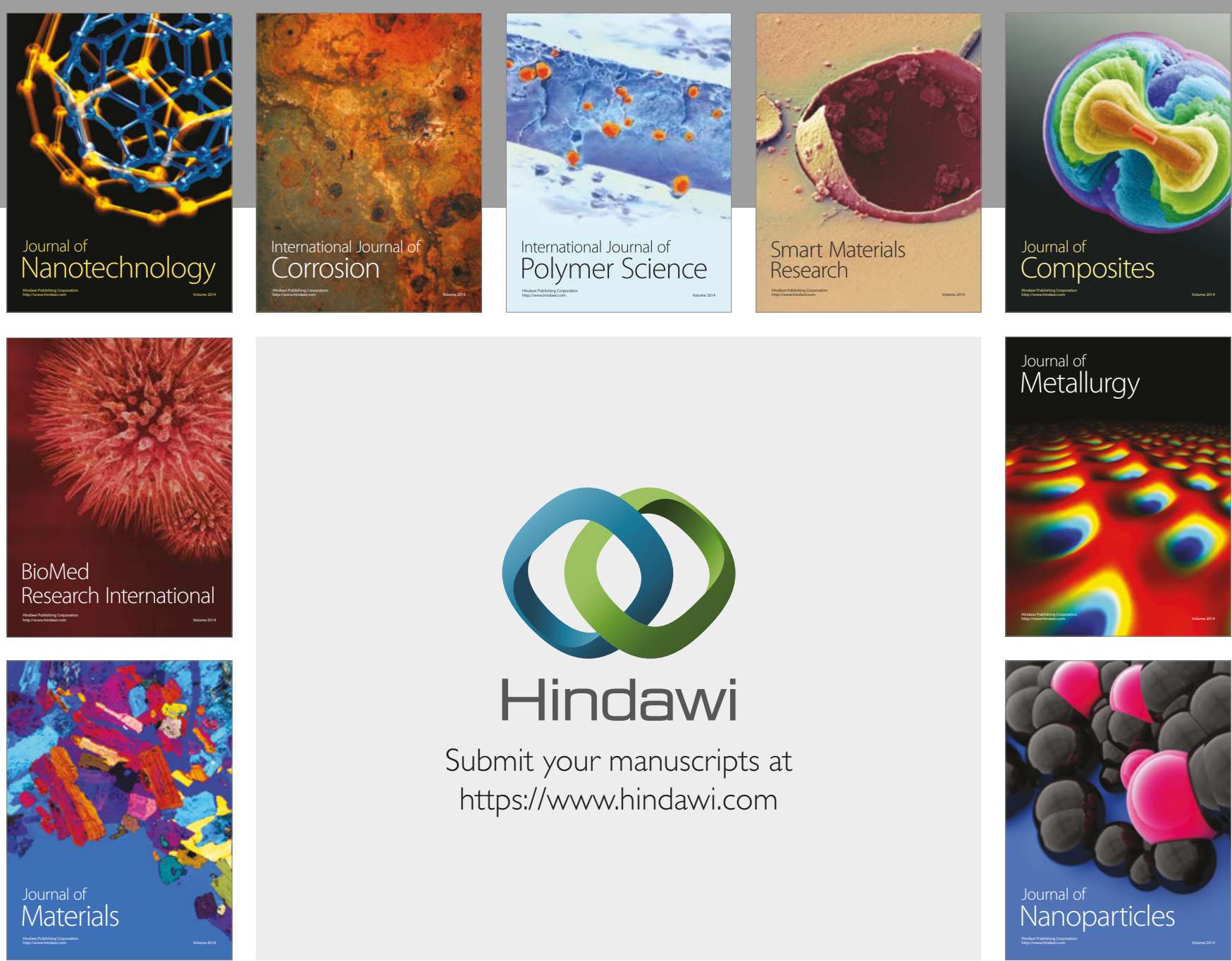

\section{Hindawi}

Submit your manuscripts at

https://www.hindawi.com
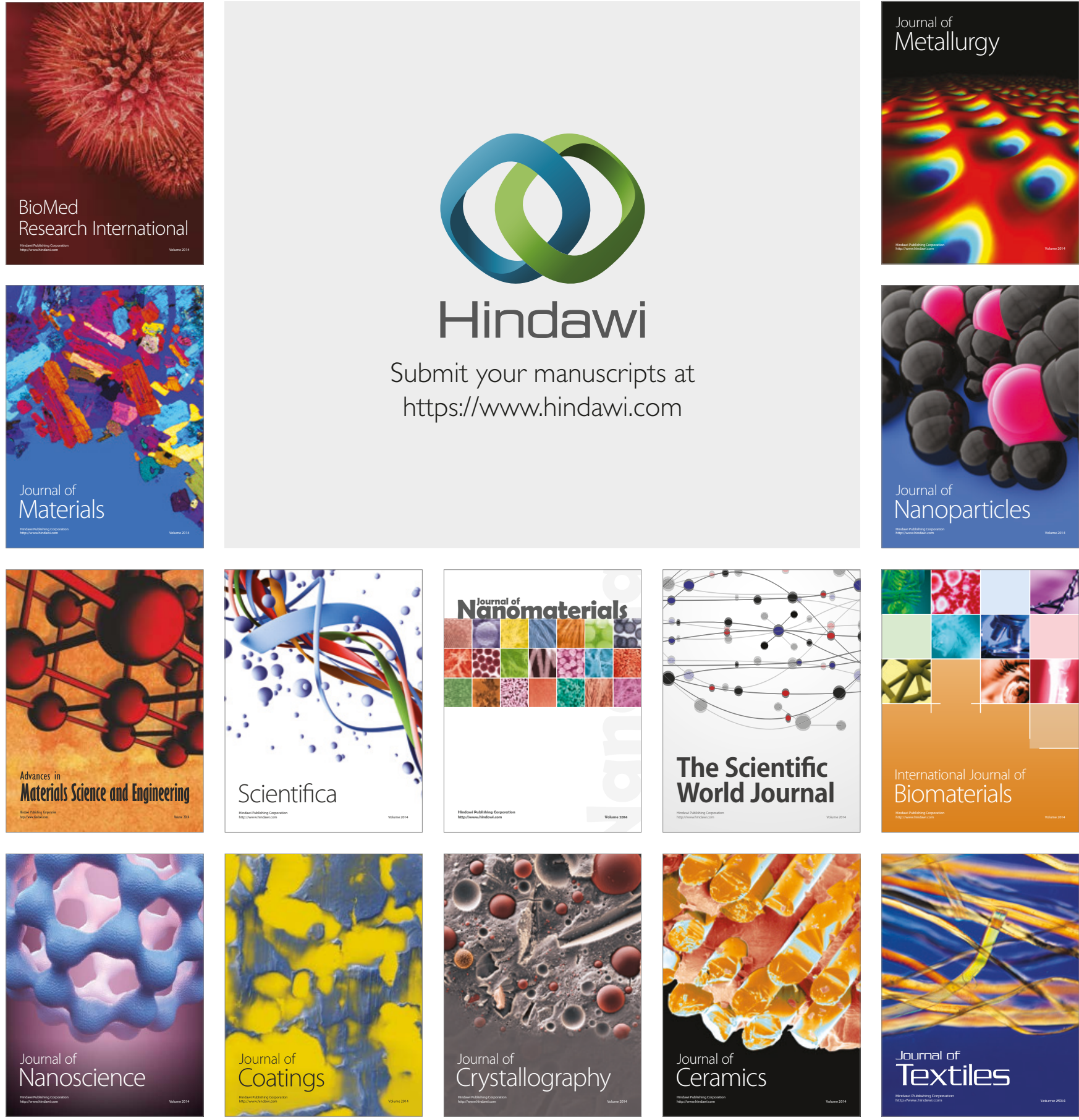

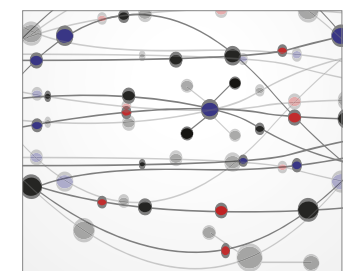

The Scientific World Journal
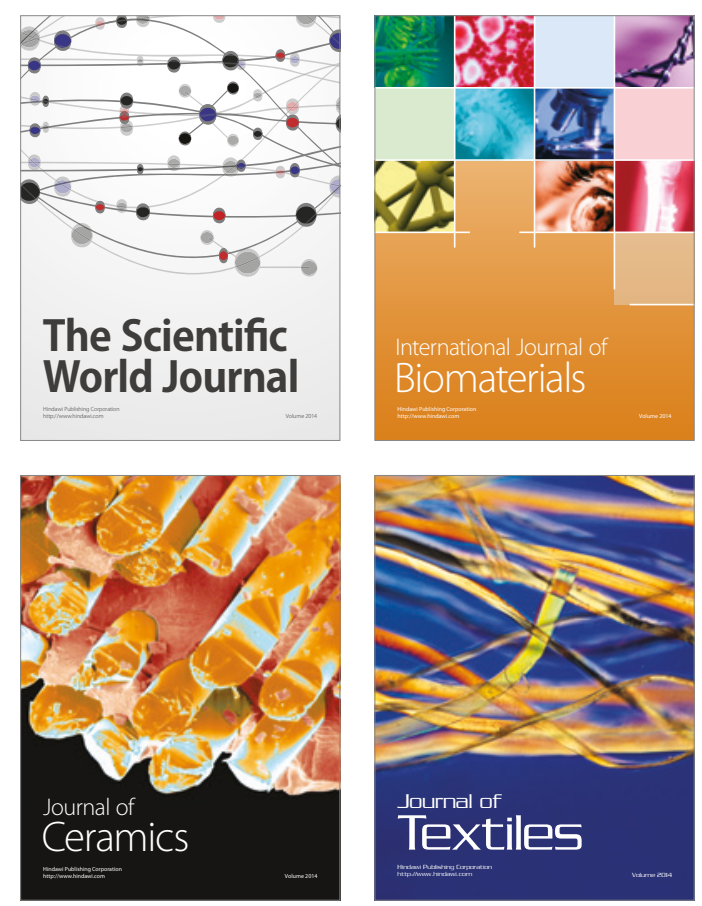